\title{
Assessment of BeiDou differential code bias variations from multi-GNSS network observations
}

\author{
S. G. Jin ${ }^{1,2}$, R. Jin ${ }^{1,3}$, and D. $\mathbf{L i}^{1}$ \\ ${ }^{1}$ Shanghai Astronomical Observatory, Chinese Academy of Sciences, Shanghai 200030, China \\ ${ }^{2}$ Department of Geomatics Engineering, Bulent Ecevit University, Zonguldak 67100, Turkey \\ ${ }^{3}$ University of Chinese Academy of Sciences, Beijing 100049, China \\ Correspondence to: S. G. Jin (sgjin@ @hao.ac.cn; sg.jin@yahoo.com)
}

Received: 19 November 2015 - Revised: 1 February 2016 - Accepted: 2 February 2016 - Published: 18 February 2016

\begin{abstract}
The differential code bias (DCB) of global navigation satellite systems (GNSSs) affects precise ionospheric modeling and applications. In this paper, daily DCBs of the BeiDou Navigation Satellite System (BDS) are estimated and investigated from 2-year multi-GNSS network observations (2013-2014) based on global ionospheric maps (GIMs) from the Center for Orbit Determination in Europe (CODE), which are compared with Global Positioning System (GPS) results. The DCB of BDS satellites is a little less stable than GPS solutions, especially for geostationary Earth orbit (GEO) satellites. The BDS GEO observations decrease the precision of inclined geosynchronous satellite orbit (IGSO) and medium Earth orbit (MEO) DCB estimations. The RMS of BDS satellites DCB decreases to about $0.2 \mathrm{~ns}$ when we remove BDS GEO observations. Zero-mean condition effects are not the dominant factor for the higher RMS of BDS satellites DCB. Although there are no obvious secular variations in the DCB time series, sub-nanosecond variations are visible for both BDS and GPS satellites DCBs during 20132014. For satellites in the same orbital plane, their DCB variations have similar characteristics. In addition, variations in receivers DCB in the same region are found with a similar pattern between BDS and GPS. These variations in both GPS and BDS DCBs are mainly related to the estimated error from ionospheric variability, while the BDS DCB intrinsic variation is in sub-nanoseconds.
\end{abstract}

Keywords. Radio science (ionospheric propagation)

\section{Introduction}

With the rapid development and wide applications of global navigation satellite systems (GNSSs), more and more constellations and stations have been constructed, including the USA's Global Positioning System (GPS), China's BeiDou Navigation Satellite System (BDS), Russia's GLObal NAvigation Satellite System (GLONASS), and the EU's Galileo, as well as other regional systems (Jin et al., 2011, 2016; Montenbruck et al., 2014). The multi-GNSS observations with multiple frequencies and multiple systems provide us more chances to monitor Earth's ionospheric variations and behaviors. The ionospheric total electron content (TEC) and electron density profile from ground-based and spaceborne multi-frequency GNSS observations can be used for ionospheric delay correction and related scientific research (Jin et al., 2006, 2007, 2013; Hernández-Pajares et al., 2011), including monitoring of Earth's ionosphere, modeling, and prediction, as well as disturbances and anomalous variations following the solar flare, geomagnetic storms, anthropogenic activities (e.g., rocket lunching and explosion), and natural hazards (e.g., earthquakes, tsunamis, and volcanic eruptions) (Calais et al., 1998; Afraimovich et al., 2010, 2013; Steiner et al., 2011; Mukhtarov et al., 2013; Occhipinti et al., 2013; Jin et al., 2010, 2014, 2015). The increasing number of onorbit GNSS satellites and operating stations have improved the temporal-spatial resolution of GNSS observations, but some biases in GNSS observables cannot be removed. The high-precision ionospheric TEC can be estimated from dualfrequency GNSS carrier phase measurement, where differential code bias (DCB) is one of the main errors, which cannot be ignored for high-precision TEC estimation and positioning applications. The receiver and satellite DCBs could be up 
to $10 \mathrm{~ns}$, i.e., more than two decades of TEC units (TECU; $1 \mathrm{~ns}$ is equivalent to $2.85 \mathrm{TECU}$ for GPS pseudo-range on L1 and L2). Due to the dispersion effect of the Earth's ionosphere, GNSS DCB could be estimated with dual-frequency observations instead of high-cost instrument calibration.

Recently, various algorithms have been developed to estimate GNSS DCBs based on multi-frequency measurements (Jin et al., 2012). Excluding the common method by setting the DCB as an unknown parameter during the GNSS TEC estimation (Mannucci et al, 1998; Schaer, 1999; Jin et al., 2012; Ma et al., 2014), the satellite and receiver DCBs can also be estimated using the IONOLAB-BIAS method with lower computation loads by taking advantage of global ionospheric maps (GIMs) (Arikan et al., 2008; Montenbruck et al., 2014). Some other optimization methods have been proposed, such as optimization by adding ionospheric residual parameters and DCB estimation based on regional high-precision ionospheric model (Keshin, 2012; Li et al., 2014). In addition, satellite-receiver geometry changes and the minimization of standard deviation of TEC have also been used to determine TEC and DCB (Ma and Maruyama, 2003; Hong et al., 2008). All of these methods are based on two basic assumptions. One is that the total electron contents in the ionosphere are concentrated in a single thin layer at $300-500 \mathrm{~km}$ altitude corresponding to maximum electron density height, i.e., the single-layer model. It is reasonable and convenient under most situations for TEC modeling and DCB estimation, except for anomalous ionospheric activities when the ionospheric variations are not homogeneous regionally. Note that DCB could also be derived from three- or four-dimensional tomography without single-layer assumption; however, it requires larger computation and is only suitable for regions with dense GNSS networks (Mitchell and Spencer, 2003). The other assumption is the zero-mean condition, which is used to estimate satellite and receiver DCBs, respectively. A constant bias will be introduced into DCB results under this condition. However, it will not affect the accuracy of TEC estimation, because the biases induced by the zero-mean condition are opposite for satellite and receiver DCBs. Generally, the results from these methods have good agreement with each other, and the GPS DCB is stable, especially for the satellite DCB, whose root mean square (RMS) is subnanosecond when there is no hardware adjustment. However, the estimated DCB results may contain error induced by the artificial assumptions, especially when the realistic ionosphere deviates from the normal state. With the appearance of more and longer GPS DCB time series, the variability in GPS DCB has been discussed. For example, day-to-day variation, annual periodic variations, and long-term trends in the satellites' DCB series have been reported (Zhang et al., 2014). The long-term trend of GPS satellite DCB has proved to be related to the zero-mean condition (Zhong et al., 2016); however, it is still a debate, and may be related to ionospheric daily and annual variations. Compared to satellite DCBs, the receiver DCBs are less stable, and their magni-

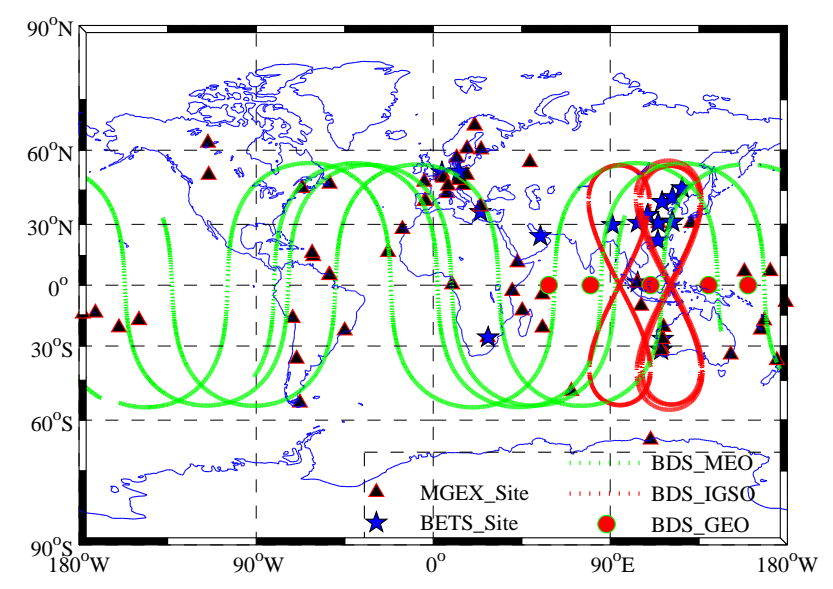

Figure 1. BDS satellite sub-trajectories for one day (15 February 2014) and the location of BDS continuously operating tracking stations from the MGEX and BETS network.

tudes and variations show large differences for different types of receivers. The receiver DCB seems to be related to the temperature around antennas (Coster et al., 2013; Yasyukevich et al., 2015; Themens et al., 2015). Besides its intrinsic variations, the estimated DCB series includes the signal associated with ionospheric anomalous variations and other errors, such as the multipath effect and the high-order effect of ionospheric delay, that are ignored in traditional TEC and DCB estimation, since non-modeling errors will affect the DCB estimation (Petrie et al., 2011). Therefore, the difference between DCB intrinsic variations and estimation errors should be considered with caution when we discuss the variation characteristics of DCB, particularly for different GNSSs.

The BeiDou Navigation Satellite System (BDS) has been constructed covering the Asia-Pacific region; it includes five geostationary Earth orbit (GEO) satellites, four medium Earth orbit (MEO) satellites, and five inclined geosynchronous satellite orbit (IGSO) satellites, shown in Fig. 1 (CNSO, 2013). A number of continuously operating BDS tracking station networks have been built, such as the Chinese BeiDou Experimental Tracking Stations (BETS) and the Multi-GNSS Experiment (Montenbruck et al., 2014). As we know, BDS measurements contain DCB errors, which affect the TEC estimation significantly. Therefore, BDS DCB and its behavior features should be evaluated before we use BDS for high-precision ionospheric modeling and applications. A number of BDS/GPS tracking stations have been operating for more than 2 years; this provides us with an opportunity to investigate the variation in the BDS DCB and discuss factors in effects on BDS DCB estimation. In this paper, the BDS DCB variation characteristics are investigated and assessed from multi-GNSS network measurements obtained by the BETS and MGEX tracking stations. 


\section{Data and methods}

\subsection{BETS and MGEX observations}

Currently, the BDS is available for regional service (CNSO, 2013). The 14 on-orbit BDS satellites' sub-trajectories are shown in Fig. 1. Although the global BDS constellation is still being expanded on, better signal coverage is available in the Asia-Pacific region from the five GEO and five IGSO satellites. At the end of 2012, the China Navigation Satellite Office publicly released interface control documents (ICDs) related to open service signal B1I and B2I in December 2013 (CNSO, 2013). BDS signals will become available for worldwide users by around 2020. More and more stations equipped with BDS receivers are being built for high-precision GNSS applications. In this paper, BDS and GPS observations collected by the BETS and MGEX stations equipped with multi-GNSS receivers during 2013-2014 are used to estimate the DCB. The locations of 20 BETS stations (marked with triangles) and 63 MGEX stations (marked with stars) are shown in Fig. 1. Most of the stations are located in the Asia-Pacific region, where the BDS GEO and IGSO satellite signals are available. Unlike GPS, the number of BDS observations has a large difference for satellites on different orbits. The BDS satellite $\mathrm{C} 05$ provides us the most abundant measurement since it is available at all times for most of the stations in Fig. 1. There was an increase in the number of stations around the middle of 2014 due to the addition of 10 new stations in the Asia-Pacific region by Geoscience Australia (http://igs.org/mgex), which increased the number of BDS GEO and IGSO observations greatly. As most of the BETS stations did not upload BDS data in 2004, there are fewer BDS observations from BETS. C30 observations are excluded in our analysis because this satellite is unavailable since the middle of 2013 with suspecting clock problems (Hauschild et al., 2012). In addition, G06, G09, G27, and G30 are also unavailable for a few days due to replacement of satellites, and these pseudo-random noise (PRN) numbers are assigned for different satellites during this period (ftp://ftp.unibe.ch/aiub/BSWUSER50/GEN/ SATELLIT.I08), which should be considered carefully when we assess 2 years of DCB estimations. Satellites are marked with space vehicle number (SVN) instead of PRN number in the following in order to avoid mixing of different satellite DCB series. For the ionospheric pierce point (IPP) location of the BDS line of sight (LOS), the approximate station coordinates are used from observation files and the precise orbit products are provided by the Wuhan University GNSS Research Center and the GeoForschungsZentrum (GFZ). For GPS LOS IPP locations, the precise ephemeris is used from the International GNSS Service (IGS).

\subsection{DCB estimation based on GIMs}

As we know, the slant TEC ( $S$ TEC) along the LOS can be expressed as the following equation, ignoring the high-order effect of the ionosphere (Jin et al., 2008):

$$
\begin{aligned}
S T E C & =\frac{f_{1}^{2} f_{2}^{2}}{40.3\left(f_{1}^{2}-f_{2}^{2}\right)}\left(L_{1} \lambda_{1}-L_{2} \lambda_{2}-\left(\lambda_{1} N_{1}-\lambda_{2} N_{2}\right)\right. \\
& \left.-\left(d_{\mathrm{r} 1}-d_{\mathrm{r} 2}+d_{1}^{\mathrm{s}}-d_{2}^{\mathrm{s}}\right)+\varepsilon_{L 12}\right) \\
& =\frac{f_{1}^{2} f_{2}^{2}}{40.3\left(f_{2}^{2}-f_{1}^{2}\right)}\left(P_{1}-P_{2}\right. \\
& \left.-\left(d_{\mathrm{r} 1}-d_{\mathrm{r} 2}+d_{1}^{\mathrm{s}}-d_{2}^{\mathrm{s}}\right)+\varepsilon_{P 12}\right),
\end{aligned}
$$

where the $f$ represents the carrier phase frequencies, $L$ and $P$ are the carrier phase and pseudo-range measurements, respectively; $N$ is the phase ambiguity; $d_{\mathrm{r}}$ and $d^{\mathrm{s}}$ are the internal hardware delay of the receiver and satellite, respectively; $\varepsilon_{L 12}$ and $\varepsilon_{P 12}$ are the other non-modeling residuals, such as multipath effect and measurement noise; and the subscripts 1 and 2 stand for the frequency numbers. Usually, $d_{\mathrm{r} 1}-d_{\mathrm{r} 2}$ and $d_{1}^{\mathrm{s}}-d_{2}^{\mathrm{s}}$ are defined as receiver and satellite DCBs, respectively. In order to reduce the amount of calculation and complexity, we use the TEC value with temporal-spatial interpolation from GIMs produced by the Center for Orbit Determination in Europe (CODE) instead of TEC estimation by means of spherical harmonic expansion or trigonometric function in GNSS ionospheric modeling. A modified singlelayer mapping function (MSLM), as in Eq. (2), is used to convert the interpolated vertical $v$ TEC to the slant STEC (Jin et al., 2004).

$$
\begin{aligned}
S T E C & =\frac{v \text { TEC }}{\cos \left(z^{\prime}\right)} \sin \left(z^{\prime}\right) \\
& =v \text { TEC } \frac{R_{\mathrm{e}}}{R_{\mathrm{e}}+\operatorname{Ion} H} \sin (\alpha z),
\end{aligned}
$$

where $R_{\mathrm{e}}$ is the radius of the Earth; Ion $H$ is the assumed single-layer ionosphere height; $z^{\prime}$ and $z$ are the zenith angles of the LOS at the receiver and IPP, respectively; and $\alpha$ is a correction factor to reduce to the mapping function error, especially for observations with low satellite angles. In our processing, Ion $H$ is set as $506.7 \mathrm{~km}$ and $\alpha$ is set as 0.9782 for converting between slant TEC and vertical TEC, which are consistent with CODE for computing the GIMs (http://www.aiub.unibe.ch). The MSLM with parameters as above is an almost optimal approximation to the Chapman profile mapping function that could reflect the actual situation generally (Schaer, 1999). GNSS receiver DCB $\left(\mathrm{DCB}_{\mathrm{r}}\right)$ plus satellite $\mathrm{DCB}\left(\mathrm{DCB}^{\mathrm{s}}\right)$ could be derived by differencing the biased STEC from GNSS dual-frequency observations and GIMs. As shown in Eq. (1), both dual-frequency carrier phase and pseudo-range measurements are used for $\mathrm{DCB}$ estimation. Here, the latter is chosen as the ionospheric observables for our DCB estimation to avoid errors induced by cycle slip repairing and phase ambiguity.

In order to assess the noise level of GIM-derived DCB, the scatters of $\mathrm{DCB}_{\mathrm{r}}$ and $\mathrm{DCB}^{\mathrm{s}}$ with different elevation angles 


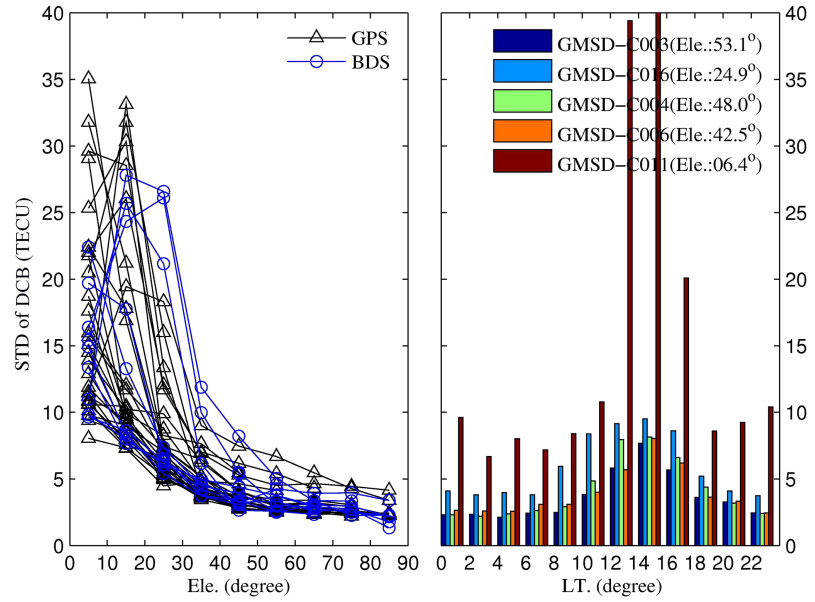

Figure 2. Left panel: standard deviation of DCB with different elevation angles based on GMSD BDS and GPS observations during 1-7 January 2014. Right panel: standard deviation of DCB with different local times based on GMSD BDS GEO observations during 1-7 January 2014.

and local times are presented in Fig. 2. Obviously, the standard deviation (SD) of DCBs that have a low elevation angle and are taken at 12:00-16:00 local time (LT) are higher, which should be related to the mapping function error induced by the low elevation angle, multipath effect, and ionospheric horizontal gradient, as well as the high RMS of the GIM during the local afternoon. The SD of the BDS DCB is similar to that of the GPS, as shown in the left panel in Fig. 2, although the elevation dependence of the BDS pseudo-range variations has been confirmed (Wanninger and Beer, 2015). After inter-frequency difference, the elevationdependent code pseudo-range variations in BDS observations have almost no influence. LT effects could be seen directly with BDS GEO observations from quasi-invariance IPP locations. This effect should be related to the lower precision of GIMs in the highly dynamic LT sectors (such as the post-noon sector). However, the LT effect is weaker than the effect of satellite elevation angles, as shown in the right panel of Fig. 2. Note that the satellite elevation angle of C011 is 6.4 $4^{\circ}$. Standard deviations of DCB corresponding to C011 are larger in almost all LTs. It is necessary to reduce the noise of GIM-derived DCB before DCB estimation. As an example, Fig. 3 shows the daily mean value of DCB corresponding to GMSD-C003, GMSD-C005, GMSD-C013, and GMSD-G063. Daily mean values of DCB from all observations with excluding measurements during 12:00-16:00 LT and low satellite elevation angle measurements (the threshold is $20^{\circ}$ here) are presented from left to right in Fig. 3 . The DCB noise is reduced dramatically with daily averaging. After removing data that are from 12:00 to 16:00 LT and that have low elevation angles, the daily DCB time series are more stable (lower daily SD) when compared to DCB series derived from all observations, especially for MEO ob-

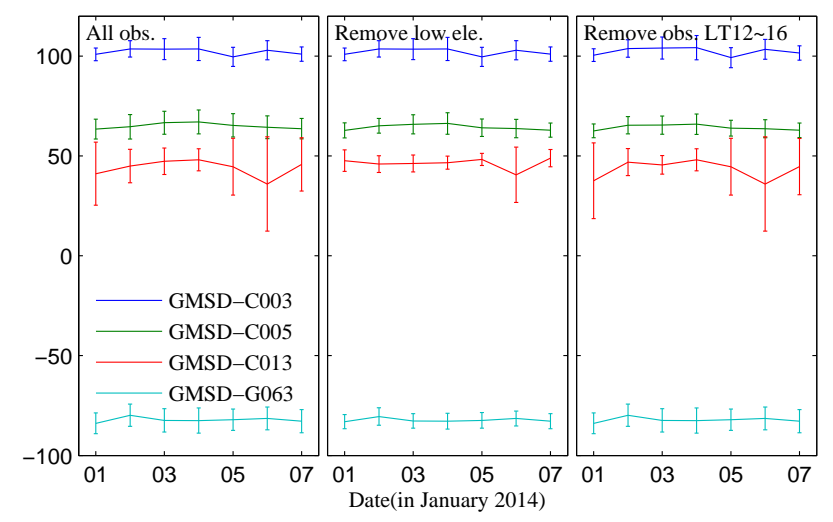

Figure 3. Daily DCBs corresponding to GMSD-C003 (blue line), GMSD-C005 (green line), GMSD-C013 (red line), and GMSDG063 (cyan line) during 1-7 January 2014. The vertical bars represent for the daily standard deviation.

servations. As shown by the SD difference with different data sets, the daily SD of DCB does not decrease dramatically after removing measurements during 12:00-16:00 LT. With the consideration of reducing noise and more redundant observations, a $20^{\circ}$ elevation angle threshold is selected and a daily mean value of all available $\mathrm{DCB}^{\mathrm{s}}$ is used to separate satellite and receiver DCB during our processing.

After extracting clean DCB, a zero-mean condition $\left(\overline{\mathrm{DCB}^{\mathrm{S}}}=0\right)$ is adopted to separate the receiver and satellite DCBs. A bias, i.e., mean value of all satellites daily DCB solutions, is clearly introduced into both the satellite and receiver DCB, as shown in Eq. (3). Here the subscript $r$ stands for the receiver number, and the superscript $s$ stands for the satellite number. There is no difference for ionospheric correction and other applications, because the biases will be eliminated when we compute the total effect of instrument biases. Furthermore, the mean value of all satellites' DCB is constant when no satellite hardware is replaced or on-orbit adjustments are made. The zero-mean condition has no effect on DCB variations.

$$
\begin{aligned}
& \sum_{i=1}^{n} \mathrm{DCB}_{\mathrm{r}}^{\mathrm{s}_{i}}=n \mathrm{DCB}_{\mathrm{r}}+\sum_{i=1}^{n} \mathrm{DCB}^{\mathrm{s}_{i}} \\
& \mathrm{DCB}_{\mathrm{r}}=\frac{\sum_{i=1}^{n} \mathrm{DCB}_{\mathrm{r}}^{\mathrm{s}_{i}}}{n}-\overline{\mathrm{DCB}^{\mathrm{s}}} \\
& \left(\overline{\mathrm{DCB}^{\mathrm{s}}}=\frac{\sum_{i=1}^{n} \mathrm{DCB}^{\mathrm{s}_{i}}}{n}\right) \\
& \mathrm{DCB}^{\mathrm{s}_{i}}=\mathrm{DCB}_{\mathrm{r}}^{\mathrm{s}_{i}}-\frac{\sum_{i=1}^{n} \mathrm{DCB}_{\mathrm{r}}^{\mathrm{s}_{i}}}{n}+\overline{\mathrm{DCB}^{\mathrm{s}}}
\end{aligned}
$$

where $\mathrm{DCB}^{\mathrm{s}}$ and $\mathrm{DCB}_{\mathrm{r}}$ are the $\mathrm{DCB}$ of satellite and receiver, respectively, and $n$ is the number of available satellites for each system. If a satellite hardware replacement or adjustment occurs, a zero-mean condition correction is needed to 


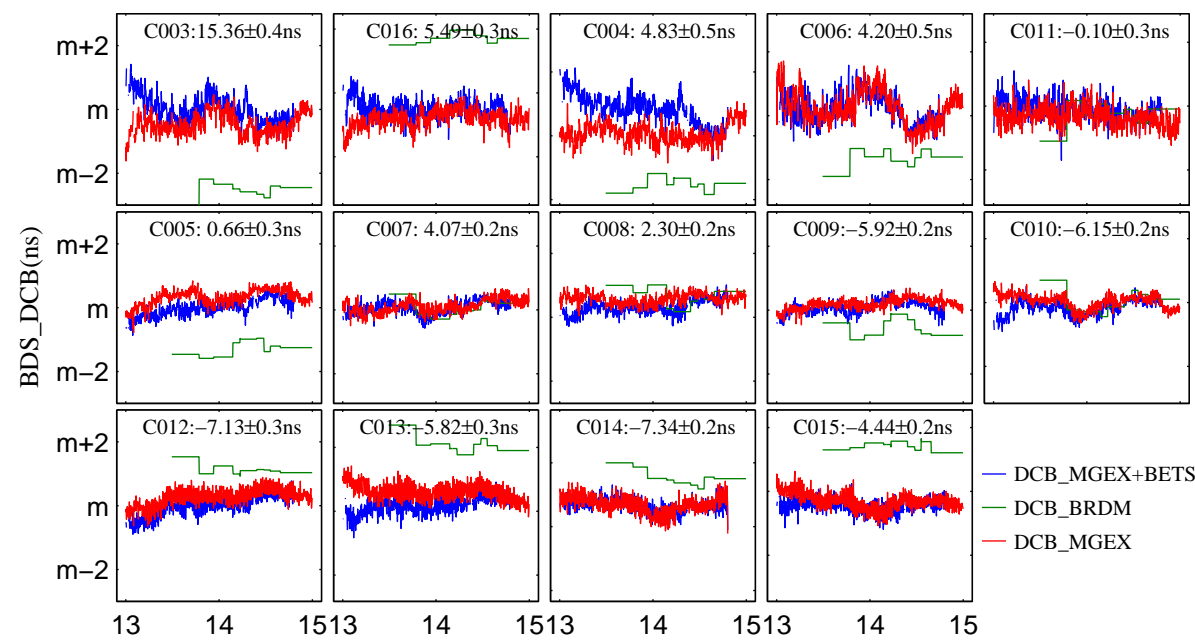

Figure 4. DCB (C2I-C7I) variations in BDS satellites during 2013-2014. The blue, cyan, and red lines are the DCBs estimated from BETS and MGEX BDS data, derived from broadcast navigation messages and provided by the MGEX website, respectively. Here " $m$ " in the vertical axis is the 2-year mean value of corresponding satellite DCBs, while the 2-year mean value and root mean square (RMS) (DCB_MGEX + BETS) are marked for each satellite. The horizontal axis ticks 13, 14, and 15 corresponding to 2013, 2014 , and 2015.

remove the jumps in the DCB series induced by the zeromean condition. Moreover, the zero-mean condition will also be changed under the situation that not all satellites are available for the GNSS network. The effect of the zero-mean condition is equivalent to a hardware change that changes the DCB of unavailable satellites to zero in corresponding days.

$$
\begin{aligned}
& \mathrm{DCB}_{\text {cur }}^{s_{j}}=\mathrm{DCB}_{\text {pre }}^{s_{j}}+d_{j}, \overline{\mathrm{DCB}}_{\text {cur }}=\overline{\mathrm{DCB}}_{\text {pre }}+\frac{d_{j}}{n_{\text {pre }}} \\
& \mathrm{DCB}_{\mathrm{r}, \text { cur, correct }}=\frac{\sum_{i=1}^{n} \mathrm{DCB}_{\text {rcur }}^{\mathrm{s}_{i}}}{n_{\text {pre }}}-\overline{\mathrm{DCB}}_{\text {pre }}-\frac{d_{j}}{n_{\text {pre }}} \\
& =\mathrm{DCB}_{\mathrm{r}, \text { cur }}-\frac{d_{j}}{n_{\text {pre }}} \\
& \mathrm{DCB}_{\text {cur, correct }}^{\mathrm{s}_{i}}=\mathrm{DCB}_{\text {cur }}^{\mathrm{s}_{i}}+\frac{d_{j}}{n_{\text {pre }}},(i \neq j)
\end{aligned}
$$

$\mathrm{DCB}^{s_{j}}$ cur $=\mathrm{DCB}^{s_{j}}$ pre $+d_{j}=\mathrm{DCB}_{\mathrm{rpre}}-\mathrm{DCB}_{\mathrm{r}, \text { pre }}+d_{j}$ $=\mathrm{DCB}_{\mathrm{r} \text { cur }}^{s_{j}}-\mathrm{DCB}_{\mathrm{r}, \text { pre }}+\frac{d_{j}}{n_{\text {pre }}}$

$d \mathrm{DCB}=\mathrm{DCB}_{\mathrm{r}}^{s_{j}}{ }_{\text {cur }}-\mathrm{DCB}_{\mathrm{rpre}}^{s_{j}}=\frac{n_{\text {pre }}-1}{n_{\text {pre }}} d_{j}$

$d=\frac{n_{\text {pre }}}{n_{\text {pre }}-1} d \mathrm{DCB}$

Given that the $\mathrm{DCB}^{s_{j}}$ is changed with an increment of $d_{j}$, the correct DCB could be derived from Eqs. (4) and (5). Here subscript "cur" and "pre" correspond to the current and previous days, respectively. After correction with Eq. (4), $\operatorname{DCB}^{\mathrm{s}_{i}}(i \neq j)$ will not be affected by the jump induced by $\mathrm{DCB}^{s_{j}}$ variations; that is, the value of $\sum_{i=1}^{n} \mathrm{DCB}^{s_{i}},(i \neq j)$ remains unchanged.

\section{Results and discussion}

In this section, the variations in BDS DCBs are estimated and investigated with 2 years of observations from the BETS and MGEX networks during 2013-2014. In order to assess BDS DCB variations, GPS DCBs derived from these BDS stations' measurements are also compared and discussed. The variation characteristics of different GNSS systems and different satellite orbits are described and analyzed below.

\subsection{BDS/GPS satellite DCB variations}

Figures 4 and 5 present the DCB time series of 14 BDS (C2IC7I) and 27 GPS (C1W-C2W, i.e., P1-P2) satellites that are extracted from BETS and MGEX observations during 2013 2014. Here the missing specific satellite-observation-induced jumps in DCB series are removed with Eqs. (4) and (5). The DCB series from the broadcast ephemeris timing group delay (TGD) are normalized with zero-mean conditions and GPS P1 C1 biases have also been corrected in order to ensure that all DCB series are in the same datum. For BDS, the estimated DCB series derived from MGEX and BETS observations agree well with the values released by MGEX but have relatively large differences (within $\pm 3 \mathrm{~ns}$ ) with DCBs determined with TGDs in broadcast ephemeris. The cause of deviation in the BDS TGDs is not clear at present but may be due to the effects of differences between the B3I and B3-Q component or incorporation of antenna offsets (Montenbruck et al., 2014). The RMSs of BDS-GEO DCBs are $0.3-0.5 \mathrm{~ns}$, which is relatively high when compared to the RMSs of BDS-MEO and IGSO DCBs, i.e., 0.2$0.3 \mathrm{~ns}$, while the RMSs of GPS satellites DCBs are around $0.1-0.2 \mathrm{~ns}$, except for G061 and G063. Obvious jumps are 


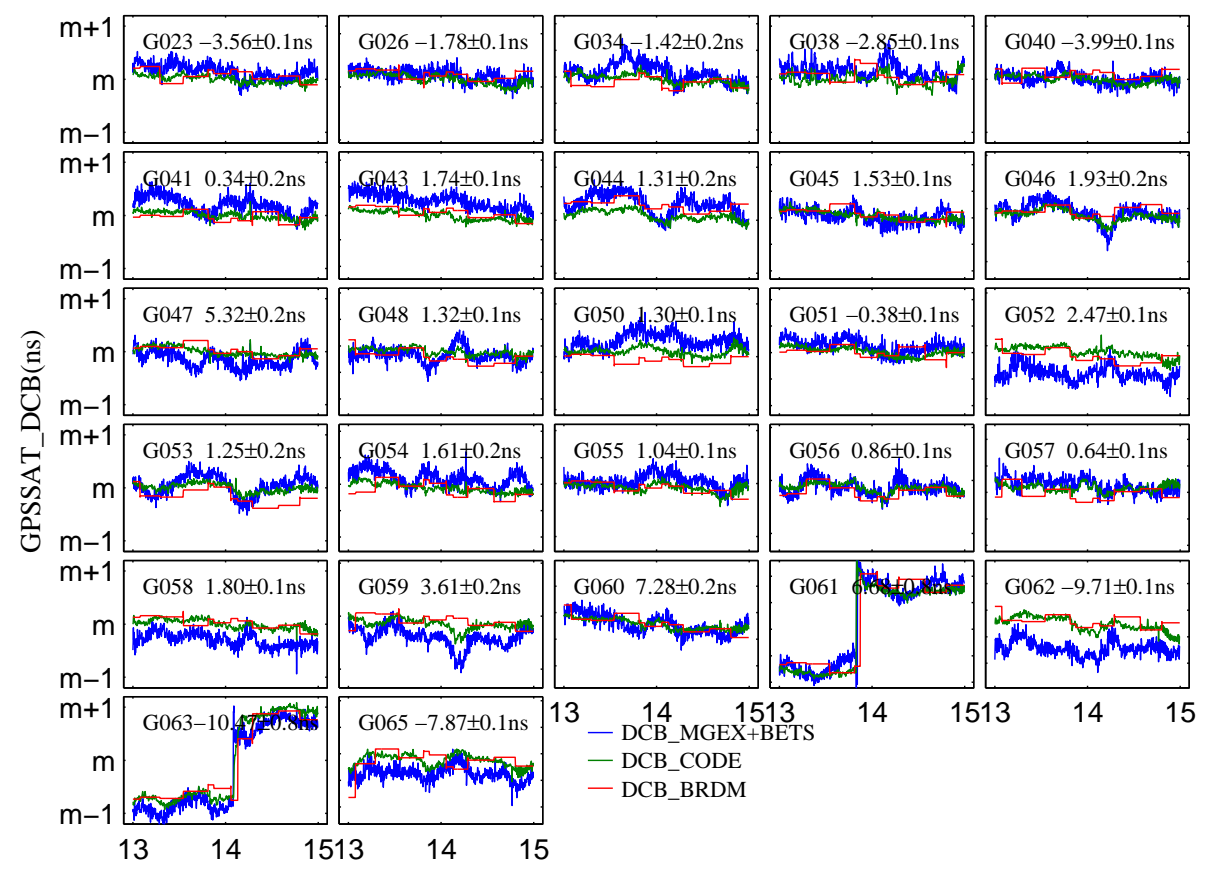

Figure 5. DCB (C1W-C2W) variations in GPS satellites derived from BETS and MGEX data based on GIMs. The blue, cyan, and red lines are the results from BETS and MGEX BDS data, broadcast navigation messages, and CODE, respectively.

recorded by CODE DCB series, broadcast TGD, and our estimated results for these two satellites. The sudden jumps are related to satellite maneuvers reported by GPS NANU 2013061 and 2014006 (http://www.navcen.uscg.gov). As we know, the locations of BDS GEO are quasi-invariant in the Earth-centered, Earth-fixed (ECEF) coordinate system. The arc mean of the difference in the observed TEC and interpolated TEC based on GIMs is more sensitive to the errors, such as those of GIMs and mapping function, because errors from GIMs and mapping function will not be attenuated dramatically by the average of the arc observations but induce a constant bias for one arc, if the location of IPP is invariant - that is, the azimuth and elevation angles of LOS remain almost constant. The lower stability of BDS-GEO DCB series is more likely related to the larger estimation error from noisier BDS GEO ionospheric observations rather than its intrinsic variations in total. GPS satellite DCBs released by CODE and the broadcast GPS satellite TGDs determined by NASA JPL with lab-calibrated reference receivers are presented as a reference to assess DCBs derived from MGEX and BETS BDS stations' measurements based on GIMs. The differences among them are rather small, as shown in Fig. 5. For SVN G052, G062, G058, and G059, the DCB series have larger deviations compared to the result of broadcast TGD and CODE released values, while the mean differences do not exceed $0.5 \mathrm{~ns}$. In general, both GPS and BDS satellites' DCBs are fairly stable over 2 years. No obvious long-term trends are found in both GPS and BDS satellite DCB series.
Compared to the BDS MEO and IGSO, the RMS of GPS satellites' DCB is almost half as small as the DCB series from MGEX and BETS observations. The difference should not be dominated by the GIM modeling and mapping errors because the locations of BDS and GPS receivers are the same. The ionospheric activities and mapping errors should be similar and reduced dramatically by obtaining the daily average DCB, as shown in Fig. 2. From Eq. (3), the variation in constellation satellites DCB mean value will be included in the estimated DCB series with the zero-mean condition. Usually the mean value of constellation satellites DCB is considered as a constant; however, its variation will be also adopted in the DCB series. According to the error propagation law, the constellation satellite mean values should become more stable as the number of satellites increases. In order to distinguish the BDS DCB intrinsic variation and zero-mean condition effects, RMSs of GPS DCB series estimated from different satellites groups are compared in Fig. 6. RMSs of GPS satellites DCB corresponding to GPS_ALL, GPS_25 (with all discontinuous satellites removed), and GPS_14 (the same number of satellite as BDS) are similar. The expected RMS increase does not appear in DCB series with GPS_26 and GPS_14, indicating that the difference of the zero-mean condition effect induced by different satellites numbers is negligible. The RMSs of DCB estimated with BDS_9 decrease dramatically (down to around $0.2 \mathrm{~ns}$ ) except for $\mathrm{C} 014$ and C015 when compared to the result of BDS_ALL. This indicates that BDS MEO/IGSO DCB will be affected by the "noisier" GEO observations if all BDS observations are used 


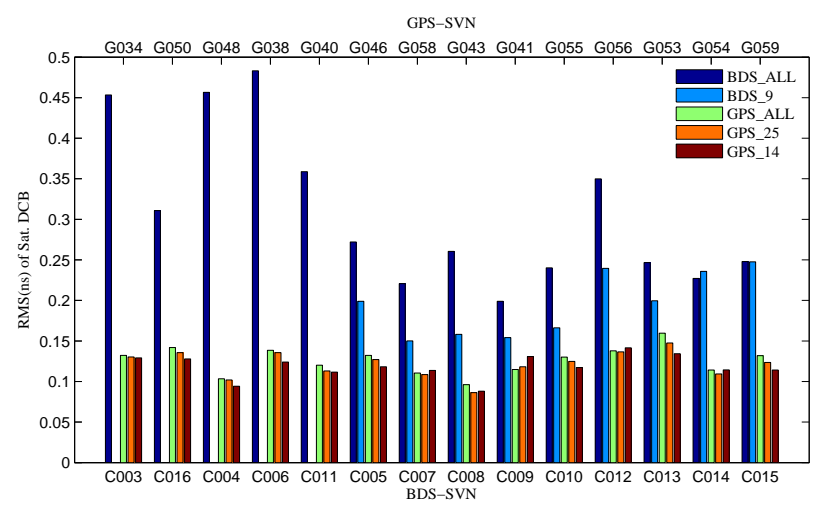

Figure 6. RMS of satellite DCB series estimated from 14 BDS satellites, 32 GPS satellites, and 14 selected satellites. "BDS_ALL' is the result based on all BDS observations, while "BDS_9" is the result with only BDS MEO/IGSO measurements. "GPS_ALL" corresponds to all orbiting GPS satellites, "GPS_25" represents available satellites except G061 and G063, and "GPS_14" represents GPS satellites with marked SVN in Fig. 1.

in DCB estimation. After removing the GEO observation, the magnitude of estimation errors in BDS and GPS DCBs is similar; however, the BDS IGSO/MEO DCB series are still less stable during 2013-2014.

Figure 7 is the monthly mean value variation in BDS and GPS satellites DCBs (here the 2-year mean values are subtracted). The 2-year mean values for individual satellite are removed for a better view of DCB variation. The annual variation signal is weak but visible, with an amplitude of around $0.3 \mathrm{~ns}$ except for the satellites in GPS orbital plane 5 and 6 . Amplitude peaks always appear around June, which indicates the variation in monthly mean value is not a random noise. It should be noted that annual variations in both BDS and GPS satellite DCBs have similar phases for satellites in the same orbital plane, while the variations are even out of phase for satellites in different orbital planes, such as GPS plane 1 and 3. As we know, F10.7 cm solar flux is a good indicator for ionospheric activities on a global scale. Here the monthly variation in F10.7 cm solar flux recorded by the Dominion Radio Astrophysical Observatory during 2013-2014 is presented in the bottom-left panel of Fig. 7 in order to show the general variation in Earth's ionosphere. Annual variations are visible in both the F10.7 series and most GPS and BDS satellites DCB series, although the phases are different for satellites in different planes. The variations in the estimated DCB series are related to the accumulation of modeling errors in the GIMs and mapping function induced by ionospheric variability along LOSs determined by specific satellite orbits; however, the relationship between ionospheric variability and DCB estimated series needs to be further investigated in the near future. It is difficult to distinguish the ionospheric induced variation in the DCB estimated from GNSS data and the intrinsic variation in DCB completely, but we could con-

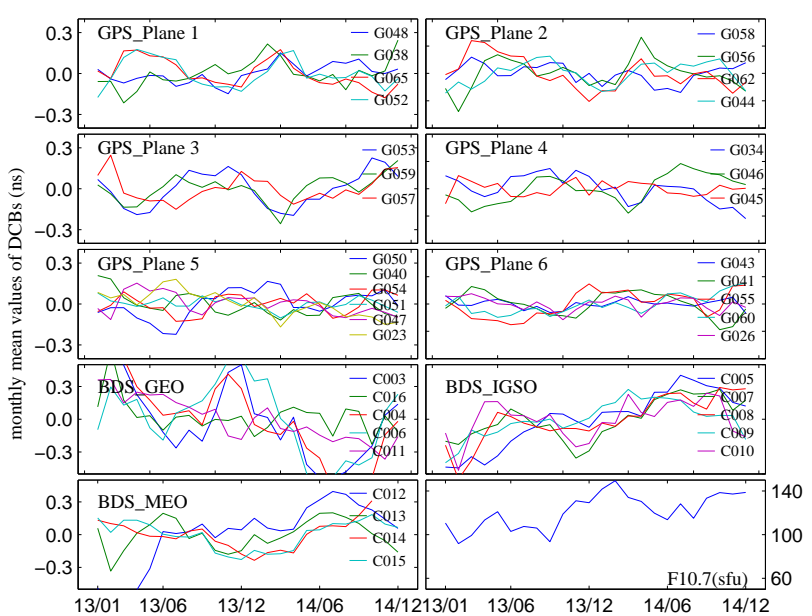

Figure 7. The variation in monthly mean values of GPS + BDS satellites DCBs. Here the 2-year mean values for individual satellites are removed for comparison. The bottom-right panel is the monthly variation in F10.7 cm solar flux provided by Natural Resources Canada (http://www.spaceweather.ca/).

firm that the DCB series include errors related to ionospheric variability and the satellite DCB should be more stable than estimated results, namely with RMS of less than $0.1-0.2 \mathrm{~ns}$ over the 2 years. The sub-nanosecond variations should be mainly related to the age of the hardware or variation in the environment surrounding the antenna, such as temperature.

Furthermore, the other frequencies' DCBs are further estimated from three frequencies' BDS observations. Figure 8 is the monthly variation in BDS satellite $\mathrm{DCB}$ corresponding to $\mathrm{C} 2 \mathrm{I}-\mathrm{C} 7 \mathrm{I}$ (B1-B2 bands), C2I-C6I (B1-B3 bands), and C7I-C6I (B2-B3 bands). It is generally similar to the situation of C2I-C7I as shown above for the other two DCB series. GEO satellite DCBs have larger RMS when compared to BDS IGSO/MEO. The monthly variations in DCB (C2I-C7I) agree well with DCB (C2I-C6I) except at the beginning of 2013 and C004, while DCB (C7I-C6I) is more stable during the 2 years. The 2-year RMS of DCBs (C7IC6I) is low, as shown in the bottom-right panel, not only for GEO but also for BDS IGSO/MEO satellites. According to Eq. (1), we know $\mathrm{DCB}=P_{1}-P_{2}-$ ionk $S$ TEC $-\varepsilon_{P 12}$, $\left(\mathrm{DCB}=d_{\mathrm{r} 1}-d_{\mathrm{r} 2}+d_{1}^{\mathrm{s}}-d_{2}^{\mathrm{s}}\right.$, and ionk $\left.=40.3\left(f_{2}^{2}-f_{1}^{2}\right) / f_{1}^{2} f_{2}^{2}\right)$. The DCB estimated error induced by ionospheric variability will be attenuated with the decrease in ionk. As shown in Table 1, the ionk is much smaller for C7I-C6I than the other pairs. It is believed that the ionospheric variability and mapping function error have lesser effect the on C7I-C6I DCB series. In other words, the higher stability of C7I-C6I DCB during 2013-2014 indicates that the variations in C2IC7I DCB are likely to be estimation biases induced by ionospheric variability and not its intrinsic variation. 


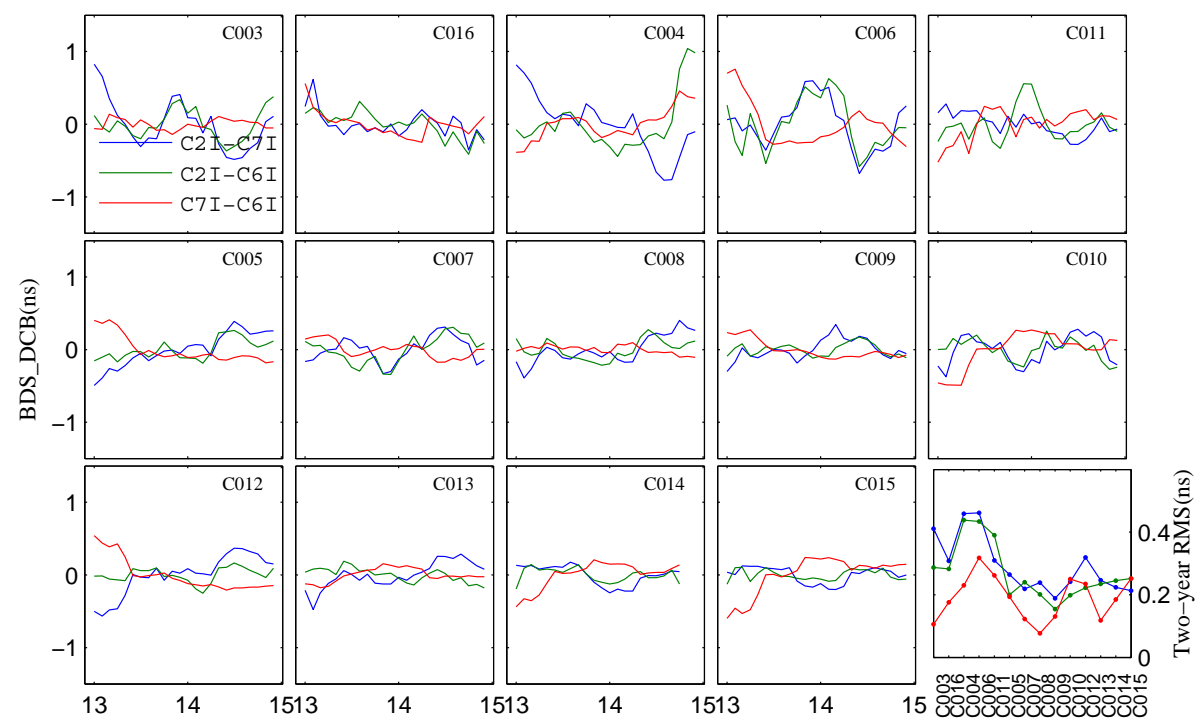

Figure 8. Monthly variations in BDS satellite DCBs for different frequency pairs. Here the 2-year mean values are subtracted. The bottomright panel presents the 2-year RMS of 14 BDS satellites' DCBs corresponding to C2I-C7I (blue), C2I-C6I(cyan), and C7I-C6I (red).

Table 1. Carrier frequencies and ionk of DCB in different carrier pairs.

\begin{tabular}{llll}
\hline & $\begin{array}{l}\text { Frequency of } \\
\text { band 1 }(\mathrm{MHz})\end{array}$ & $\begin{array}{l}\text { Frequency of } \\
\text { band 2 }(\mathrm{MHz})\end{array}$ & $\begin{array}{l}\text { ionk } \\
(\mathrm{m} / \mathrm{TECU})\end{array}$ \\
\hline CIC-C2W & 1575.42 & 1227.60 & -0.105 \\
C2I-C7I & 1561.098 & 1207.140 & -0.112 \\
C2I-C6I & 1561.098 & 1268.520 & -0.085 \\
C7I-C6I & 1207.140 & 1268.520 & -0.026 \\
\hline
\end{tabular}

\subsection{BDS/GPS receiver DCB variations}

Since most BETS and MGEX stations were not completely continuously operating in 2013-2014, 18 BDS + GPS stations with long-period continuous observations are used to estimate BDS receiver DCB variations during 2013-2014. In order to avoid the error caused by "noisier" GEO observations and satellite updates, the DCB series in Fig. 9 are based on BDS_9 and GPS_25. The jumps induced by hardware operations and zero-mean condition effects in the receiver DCB series are corrected. Similar to GPS, BDS receiver DCB RMSs are much larger than satellites ones, although their RMSs are around $0.5 \mathrm{~ns}$. Compared to GPS receiver DCBs, BDS receiver DCB series are a little less stable, which is similar to the situation of satellite DCB. The satellite DCB is obviously independent of the receivers DCB in a physics sense, while differences between BDS and GPS are almost the same as those of receivers (around $0.1 \mathrm{~ns}$ in midlatitudes). The systematic difference in the DCB series should not be due to instrument bias variations but rather the effects induced by other factors for BDS and GPS observations, such as multipath and some other non-modeling errors.
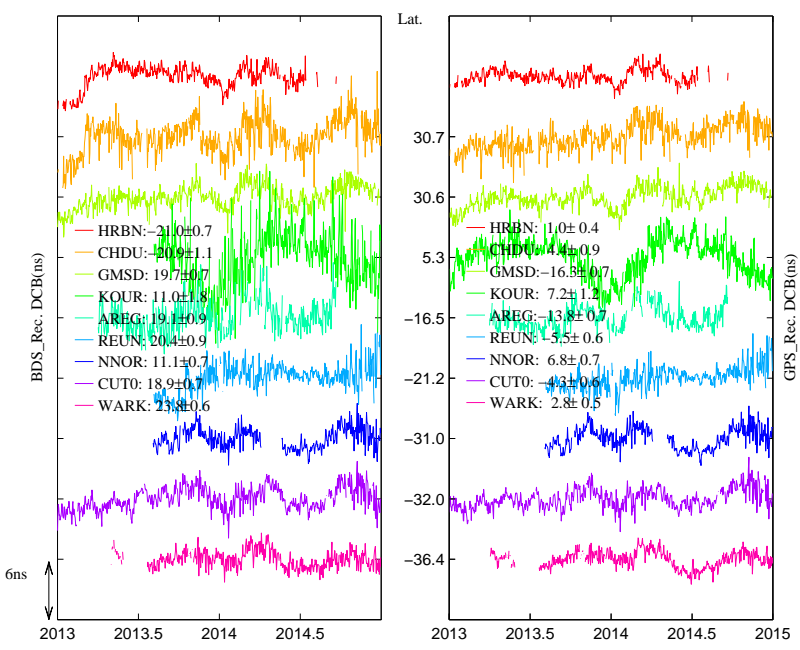

Figure 9. BDS and GPS DCB (C2I-C7I/CIC-C2W) variations in nine stations in the Asia-Pacific region.

The real variations in GPS and BDS receivers DCBs should be similar. In addition, not only GPS but also BDS receivers' DCB variations in these adjacent stations show strong correlations; however, there is no visible different variation for different antennas.

Figure 9 shows the receiver DCB variation for nine selected stations in the Asia-Pacific region during 2013-2014. The stations' latitudes are marked in the middle. We can see that the closer two stations are, the more relevant the receivers' DCB variation appears. Since the station is close to the Equator, RMSs for both BDS and GPS DCB increase, except for station CHDU. As we know, a few IGS stations are used to derive GIMs in CODE's processing, which leads 


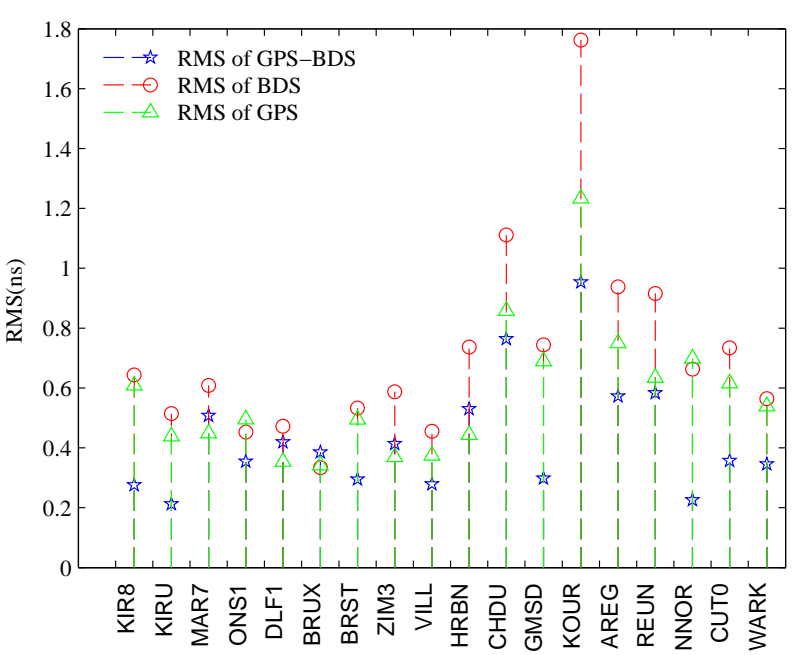

Figure 10. Two-year RMS of GPS minus BDS DCBs (C2IC7I/CIC-C2W) estimated from MGEX and BETS network observations corresponding to the 18 selected stations.

to the relatively low precision of GIM in the Asia-Pacific areas. Since GIMs could not describe the details of ionospheric variations accurately in this area and the ionosphere around the Equator is more active, GIM modeling error increases. Figure 10 presents the two RMSs between BDS and GPS receiver DCBs at 18 selected BDS stations. The RMS decreases dramatically compared to the BDS DCB RMS, except for station BRUX. The regional variation characteristics and the consistency of the estimated BDS and GPS receiver DCB variations at one station show that the GIMs-derived DCBs should be related to the GIM estimation error. When one bias is added to DCB, the magnitudes of estimated satellite and receiver DCBs errors should be similar to the zeromean condition, while the satellite DCBs are much more stable because regional GIM estimation error will be attenuated when multiple stations in different areas are used in DCB estimation.

Figure 11 shows the results of C2I-C7I, C2I-C6I, and C7I-C6I receiver DCB variations at CUT0 and GMSD, whose observations are available for almost all days during 2013 and 2014 without jumps induced by receivers' replacement and other artificial operation. Similar to satellites' DCB variations, the estimated $\mathrm{C} 2 \mathrm{I}-\mathrm{C} 7 \mathrm{I}$ receiver DCBs and $\mathrm{C} 2 \mathrm{I}-$ C6I receiver DCB series have high consistency with each other, while C7I-C6I receiver DCBs are much more stable, with a smaller RMS of around $0.3 \mathrm{~ns}$ even though these two stations (as shown in Table 1) are located in an area where the precision of GIM TEC is relatively low due to sparsity of IGS stations. The ionospheric-variability-induced error in the GIM TEC should be an important source of the BDS receiver DCB variations. As shown in Figs. 8 and 11, these effects could be greatly reduced by the carrier pairs with lower "ionk".

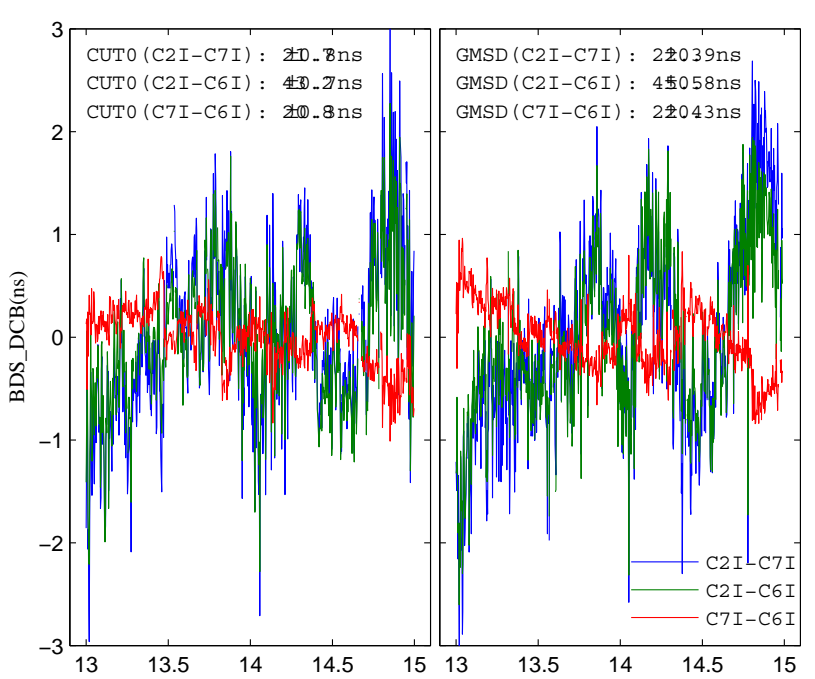

Figure 11. BDS receiver DCB variations in C2I-C7I, C2I-C6I, and C7I-C6I at BDS stations CUT0 and GMSD during 2013-2014.

\section{Summary}

In this paper, BDS and GPS satellite and receiver DCBs are estimated based on GIMs using the multi-GNSS measurements from the BETS and MGEX networks. The results show that both BDS and GPS satellite DCB RMSs are less than $0.3 \mathrm{~ns}$ - except for BDS GEO satellites, whose RMS is around $0.4-0.5 \mathrm{~ns}-$ after removing jumps induced by the zero-mean condition effects. The RMS of BDS IGSO/MEO DCBs is decreased when we remove the GEO observations. Annual DCB variations are found for satellites in the same orbital plane. The variations in the DCBs are more likely to be related to ionospheric activity and mapping function errors. The BDS receiver DCB (C2I-C7I) is less stable than GPS results (C1C-C2W) during 2013-2014, with an RMS of around $0.3-1.8 \mathrm{~ns}$. The RMS of BDS receivers' DCBs is a little larger than those of GPS even at the same station, but their trends are almost the same. Furthermore, the receivers' DCBs become "noisy" when the station's location is closer to the region with lower-precision GIMs. This is important evidence to show that GNSS-estimated DCBs contain the modeling error induced by ionospheric variability. In addition, not only satellite but also receiver DCBs in C7I-C2I are much more stable, while C2I-C7I and C2I-C6I DCB series have higher and similar variations. Ionospheric-variabilityinduced errors in GIM TEC and mapping function should be dominant factors for the fluctuation of C2I-C7I and C2I-C6I DCBs. Although it is difficult to separate the ionosphericvariability-induced errors and the intrinsic variation in DCB, both BDS satellite and receiver DCBs are rather stable. BDS observations, like GPS, can be used for ionospheric modeling. In the future, with the benefit of multi-GNSS observations, it is expected that ionospheric models with high 
temporal-spatial resolution will be able to be driven by combining BDS and GPS measurements.

Acknowledgements. The authors thank the IGS for providing MGEX data and Wuhan University for providing BETS data. This work was supported by the National Basic Research Program of China (973 Program) (grant no. 2012CB720000) and the National Natural Science Foundation of China (NSFC) project (grant nos. 11373059 and 11573052).

The topical editor, K. Hosokawa, thanks V. Demyanov and one anonymous referee for help in evaluating this paper.

\section{References}

Afraimovich, E. L., Ding, F., Kiryushkin, V. V., Astafyeva, E. I., Jin, S., and Sankov V. A.: TEC response to the 2008 Wenchuan Earthquake in comparison with other strong earthquakes, Int. J. Remote Sens., 31, 3601-3613, 2010.

Afraimovich, E. L., Astafyeva, E., Demyanov, V. V., Edemskiy, I., Gavrilyuk, N., Ishin, A., Kosogorov, E., Leonovich, L., Lesyuta, O., Palamartchouk, K., Perevalova, N., Polyakova, A., Smolkov, G., Voeykov, S., Yasyukevich, Y., and Zhivetiev, E.: A review of GPS/GLONASS studies of the ionospheric response to natural and anthropogenic processes and phenomena, Journal of Space Weather and Space Climate, 3, A27, doi:10.1051/swsc/2013049, 2013.

Arikan, F., Nayir, H., Sezen, U., and Arikan, O.: Estimation of single station interfrequency receiver bias using GPS-TEC, Radio Sci., 43, RS4004, doi:10.1029/2007RS003785, 2008.

Calais, E., Minster, J. B., Hofton M. A., and Hedlin, M. A. H.: Ionospheric signature of surface mine blasts from Global Positioning System measurements, Geophys. J. Int., 132, 191-202, 1998.

CNSO: BeiDou Navigation Satellite System Signal In Space Interface Control Document: Open Service Signal, Version 2.0, China Satellite Navigation Office, Beijing, China, 2013.

Coster, A., Williams, J., Weatherwax, A., Rideout W., and Herne, D.: Accuracy of GPS total electron content: GPS receiver bias temperature dependence, Radio Sci., 48, 190-196, 2013.

Hauschild, A., Montenbruck, O., Sleewaegen, J. M., Huisman, L., and Teunissen, P. J. G.: Characterization of Compass M-1 signals, GPS Solut., 16, 117-126, 2012.

Hernández-Pajares, M., Juan, J. M., Sanz, J., Aragón-Àngel, À., García-Rigo, A., Salazar, D., and Escudero, M.: The ionosphere: effects, GPS modeling and the benefits for space geodetic techniques, J. Geodesy, 85, 887-907, 2011.

Hong, C. K., Grejner-Brzezinska, D. A., and Kwon, J. H.: Efficient GPS receiver DCB estimation for ionosphere modeling using satellite-receiver geometry changes, Earth Planets Space, 60, E25-E28, doi:10.1186/BF03353138, 2008.

Jin, R., Jin, S., and Feng, G.: M_DCB: Matlab code for estimating GNSS satellite and receiver differential code biases, GPS Solut., 16, 541-548, 2012.

Jin, S. G., Wang, J., Zhang, H., and Zhu, W. Y.: Real-time monitoring and prediction of the total ionospheric electron content by means of GPS observations, Chinese J. Astron. Ast., 28, 331337, doi:10.1016/j.chinastron.2004.07.008, 2004.
Jin, S. G., Park, J., Wang, J., Choi, B., and Park, P., Electron density profiles derived from ground-based GPS observations, J. Navigation, 59, 395-401, doi:10.1017/S0373463306003821, 2006.

Jin, S. G., Cho, J., and Park, J.: Ionospheric slab thickness and its seasonal variations observed by GPS, J. Atmos. Sol.-Terr. Phys., 69, 1864-1870, doi:10.1016/j.jastp.2007.07.008, 2007.

Jin, S. G., Luo, O. F., and Park, P. H.: GPS observations of the ionospheric F2-layer behavior during the 20th November 2003 geomagnetic storm over South Korea, J. Geodesy, 82, 883-892, doi:10.1007/s00190-008-0217-x, 2008.

Jin, S. G., Zhu, W. Y., and Afraimovich, E., Co-seismic ionospheric and deformation signals on the 2008 magnitude 8.0 Wenchuan Earthquake from GPS observations, Int. J. Remote Sens., 31, 3535-3543, doi:10.1080/01431161003727739, 2010.

Jin, S. G., Feng, G. P., and Gleason, S.: Remote sensing using GNSS signals: current status and future directions, Adv. Space Res., 47, 1645-1653, doi:10.1016/j.asr.2011.01.036, 2011.

Jin, S. G., van Dam, T., and Wdowinski, S.: Observing and understanding the Earth system variations from space geodesy, J. Geodyn., 72, 1-10, doi:10.1016/j.jog.2013.08.001, 2013.

Jin, S. G., Jin, R., and Li, J. H.: Pattern and evolution of seismoionospheric disturbances following the 2011 Tohoku earthquakes from GPS observations, J. Geophys. Res.-Space, 119, 79147927, 2014.

Jin, S. G., Occhipinti, G., and Jin, R.: GNSS ionospheric seismology: Recent observation evidences and characteristics, Earth-Sci. Rev., 147, 54-64, doi:10.1016/j.earscirev.2015.05.003, 2015.

Jin, S. G., Qian, X. D., and Kutoglu, H.: Snow depth variations estimated from GPS-Reflectometry: A case study in Alaska from L2P SNR data, Remote Sens., 8, 63, doi:10.3390/rs8010063, 2016.

Keshin, M.: A new algorithm for single receiver DCB estimation using IGS TEC maps, GPS Solut., 16, 283-292, 2012.

Li, Z., Yuan, Y., Fan, L., Huo X., and Hsu, H.: Determination of the Differential Code Bias for Current BDS Satellites, IEEE T. Geosci. Remote., 52, 3968-3979, 2014.

Ma, G. and Maruyama, T.: Derivation of TEC and estimation of instrumental biases from GEONET in Japan, Ann. Geophys., 21, 2083-2093, doi:10.5194/angeo-21-2083-2003, 2003.

Ma, G., Gao, W., Li, J., Chen, Y., and Shen, H.: Estimation of GPS instrumental biases from small scale network, Adv. Space Res., 54, 871-882, 2014.

Mannucci, A. J., Wilson, B. D., Yuan, D. N., Ho, C. H., Lindqwister, U. J., and Runge, T. F.: Global mapping technique for GPSderived ionospheric total electron content measurements, Radio Sci., 33, 565-582, 1998.

Mitchell, C. N. and Spencer, P. S.: A three-dimensional timedependent algorithm for ionospheric imaging using GPS, Ann. Geophys.-Italy, 46, 687-696, 2003.

Montenbruck, O., Hauschild, A., and Steigenberger, P.: Differential Code Bias Estimation using Multi-GNSS Observations and Global Ionosphere Maps, J. Inst. Navig., 61, 191-201, 2014.

Mukhtarov, P., Pancheva, D., Andonov, B. and Pashova, L.: Global TEC maps based on GNSS data: 1. Empirical background TEC model, J. Geophys. Res.-Space, 118, 4594-4608, 2013.

Occhipinti, G., Rolland, L., Lognonné, P., and Watada, S.: From Sumatra 2004 to Tohoku-Oki 2011: The systematic GPS detection of the ionospheric signature induced by 
tsunamigenic earthquakes, J. Geophys. Res., 118, 3626-3636, doi:10.1002/jgra.50322, 2013.

Petrie, E. J., Hernández-Pajares, M., Spalla, P., Moore, P., and King, M. A. A review of higher order ionospheric refraction effects on dual frequency GPS, Surv. Geophys., 32, 197-253, 2011.

Schaer, S.: Mapping and predicting the Earth's ionosphere using the Global Positioning System, Geod.-Geophys. Arb. Schweiz, 59, 59 pp., 1999.

Steiner, A. K., Lackner, B. C., Ladstädter, F., Scherllin-Pirscher, B., Foelsche, U., and Kirchengast, G.: GPS radio occultation for climate monitoring and change detection, Radio Sci., 46, RS0D24, doi:10.1029/2010RS004614, 2011.

Themens, D. R., Jayachandran, P. T., and Langley, R. B.: The nature of GPS differential receiver bias variability: An examination in the polar cap region, J. Geophys. Res.-Space, 120, 8155-8175, 2015 .
Wanninger, L. and Beer, S.: BeiDou satellite-induced code pseudorange variations: diagnosis and therapy, GPS Solut., 19, 639-648, 2015.

Yasyukevich, Y. V., Mylnikova, A. A., Kunitsyn, V. E., and Padokhin, A. M.: Influence of GPS/GLONASS differential code biases on the determination accuracy of the absolute total electron content in the ionosphere, Geomagn. Aeronomy+, 55, 763$769,2015$.

Zhang, D., Shi, H., Jin, Y., Zhang, W., Hao, Y., and Xiao, Z.: The variation of the estimated GPS instrumental bias and its possible connection with ionospheric variability, Sci. China Ser. E, 57, 67-79, 2014

Zhong, J., Lei, J., Dou, X., and Yue, X.: Is the long-term variation of the estimated GPS differential code biases associated with ionospheric variability?, GPS Solut., 1-7, doi:10.1007/s10291-0150437-5, 2016. 